\title{
CAD-based path planning for 3-D line laser scanning
}

\author{
F. $\mathrm{Xi}^{\mathrm{a}, *}$, C. Shu ${ }^{\mathrm{b}}$ \\ ${ }^{a}$ Integrated Manufacturing Technologies Institute, National Research Council Canada, 800 Collip Circle, London, Ontario, Canada N6G $4 X 8$ \\ ${ }^{\mathrm{b}}$ Institute for Information Technology, National Research Council Canada, 1500 Montreal Road, Bldg. M-50, Ottawa, Ontario, Canada K1A 0R6
}

Received 25 November 1998; received in revised form 23 March 1999; accepted 7 April 1999

\begin{abstract}
The problem of path planning for 3-D line laser scanning is addressed in this article. The path under study is for a line, as opposed to a point in conventional CNC and CMM toolpath planning. While the objective of the latter case is to drive a point along a curve, that of the former case is to drive a line across a surface. To solve this problem, a path planning method is developed. First, a slicing approach is put forward to divide a part CAD model into a number of sections, since the laser scanning line is limited by the scanning width and a single scanning pass cannot cover the whole surface. Second, for each section, a scanning pass is generated to cover the section surface. To ensure a maximum coverage for each section, the surface profile is determined, and the top of the scanning window is set along the upper boundary of the surface profile. Third, combination of scanning all the sections forms a complete scan of a surface. It is shown that with the proposed method a 3-D line laser scanning process can be effectively automated. (C) 1999 Elsevier Science Ltd. All rights reserved.
\end{abstract}

Keywords: 3-D line laser scanning; Path planning method; Tool path planning

\section{Introduction}

The laser scanner has emerged as an effective non-contact tool for digitizing 3-D surfaces [1]. The newly developed 3D line laser scanner has further improved the scanning efficiency from the point laser scanner [2]. Initially, the laser scanner was mainly used for reverse engineering [3], where surface digitization data is generated and then reconstructed into a CAD model for prototype production.

Recently, the laser scanner has been utilized for part inspection [4]. Compared to the conventional coordinate measurement machine (CMM), laser scanning is noncontact (non-invasive), faster, and with a higher resolution of surface digitization. Non-contact means is required for soft or fragile object measurement. While CMM still remains an important tool for direct inspection, laser scanning is becoming an effective tool for CAD-based inspection. Direct inspection is performed by comparison between the measurement data with the specified tolerance. CADbased inspection is carried out by comparing a reconstructed model from scanning data with the original CAD model, allowing a full integration of design with inspection by including manufacturing information such as tolerance into CAD model [5].

* Corresponding author. Tel.: + 1-519-430-7062; fax: + 1-519-4307032 .

E-mail address: jeff.xi@urc.ca (F. Xi)
Basic research in laser scanning has been mainly focused on accuracy improvement. The basic principle behind the point laser scanner is triangulation. Modeling techniques have been reported in the literature for investigating the factors including incidental angle and surface reflectivity [6]. The integrated systems have been envisioned combining the touch trigger probe and the point laser scanner for both contact and non-contact measurement $[4,6]$. To improve the efficiency of the point laser scanner, the 3-D line laser scanner was created by rotating a mirror to sweep a laser beam across to form a scanning line [7]. The speckle noise associated with the 3-D line laser scanner was studied [8]. The current 3-D line laser scanner has a reasonably good accuracy applicable for manufacturing applications, such as rapid prototyping.

One of the problems in the current practice of laser scanning is that most operations are still done manually. For scanning a part, the operator needs to drive the laser scanner back and forth many times through various viewpoints. Scanning without path planning is not only time consuming, but has no active control over scanning coverage, causing either excessive (over) scanning or missing some regions (under scanning). Milroy et al. [9] tackled the problem of path planning for the 3-D line laser scanner from the point of view of reverse engineering, where the part model is unknown. The issue under their study was to find the next best viewpoint for each successive scan based on the orthogonal cross section (OCS) model. With the OCS 


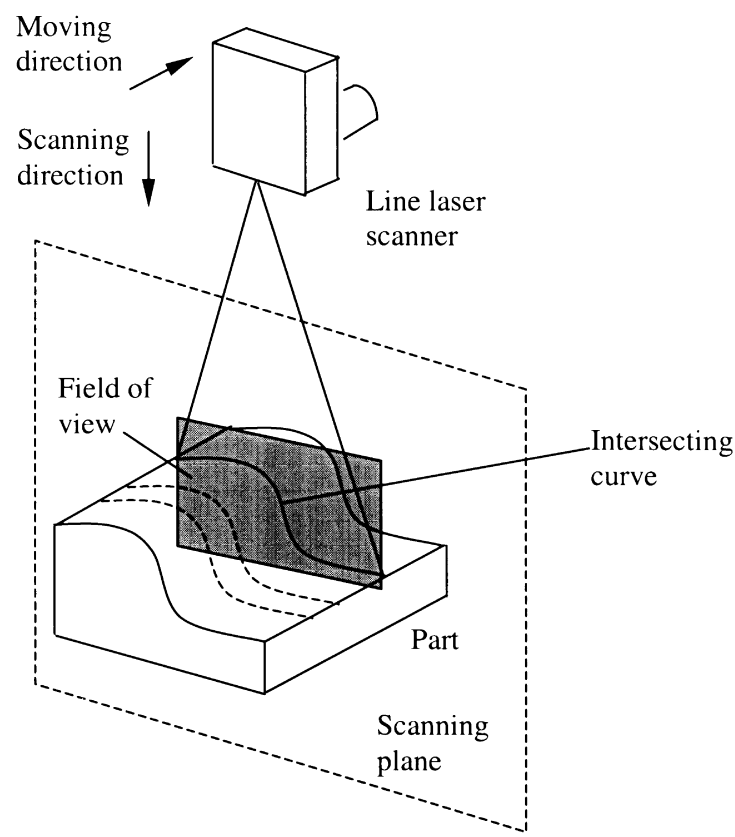

Fig. 1. 3-D line laser scanning.

model, an initial scan starts from the top and then goes to the next scan with a given step size. However, in their implementation, since the laser scanner was not mounted on a motorized turning/tilting table, viewpoints were set up
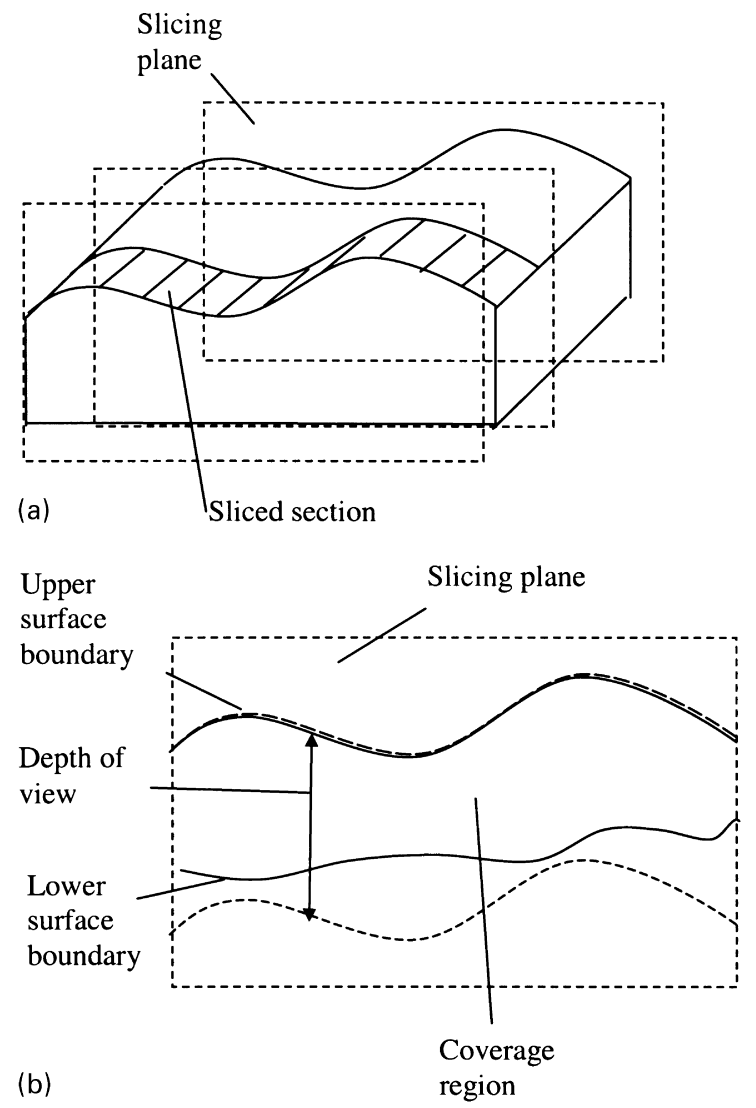

Fig. 2. (a) Slicing concept. (b) Surface boundary and coverage region. manually for each successive scan. Also, addition means, such as vision, is required in order to identify the object to be scanned and then bring the laser scanner to the initial scanning position.

In this article, we address the problem of path planning for the 3-D line laser scanning from the point of view of inspection. The nature of the problem under this study is similar to toolpath planning for $\mathrm{CNC}$ and $\mathrm{CMM}$, that is, to generate path based on CAD model. However, CNC/CMM toolpath planning is not exactly applicable to the 3-D line laser scanning path planning. The difference lies in that for the former case the objective is to drive a point along a given curve, while in the latter case is to drive a line across a given surface. The underlying issue is how to plan, based on the part CAD model, for a line to sweep through a surface with a maximum coverage. In what follows, this problem is formulated, and a solution method is presented with tests.

\section{Problem formulation}

Prior to formulating the path planning problem, a brief review on the 3-D line laser scanning is provided. As shown in Fig. 1, the scanning process can be described by a scanning plane that moves in a certain direction. In the scanning plane, there is a scanning window, called field of view (FOV), defined by scanning width $L$ and the depth of view $D$. Only the scanning image data falling within FOV (shaded area in Fig. 1) will be collected. As the scanner moves, it generates a rectangular tube volume that defines an acceptable scanning data region.

Now let us consider the surface being scanned. As shown in Fig. 1, the intersection between the scanning plane and the surface is a curve. Though the scanner allows variation in the scanning direction within FOV, a question arises as how to set the FOV relative to the intersecting curve for the purpose of maximizing the coverage for each line scanning. An intersecting curve would not be properly covered if FOV were set too high or too low. In other words, the setting of FOV has to be optimal. Furthermore, this setting is dynamic as the surface geometry changes in the direction that the scanner moves.

If the top of FOV, which is a line, is selected as a reference for optimal setting, the problem of path planning for the 3-D line laser scanning may be defined as to find a path for driving this line to follow the surface.

\section{Methodology}

Before addressing the issue of optimal setting of FOV, we need to discuss part slicing. For a given part, due to the limit of scanning width a single scanning pass may not always be able to cover the whole surface. To solve this problem, the slicing concept is introduced to divide the CAD model of the part to be scanned into a number of sections, as shown in Fig. 2(a), with each section being covered by a scan pass. 


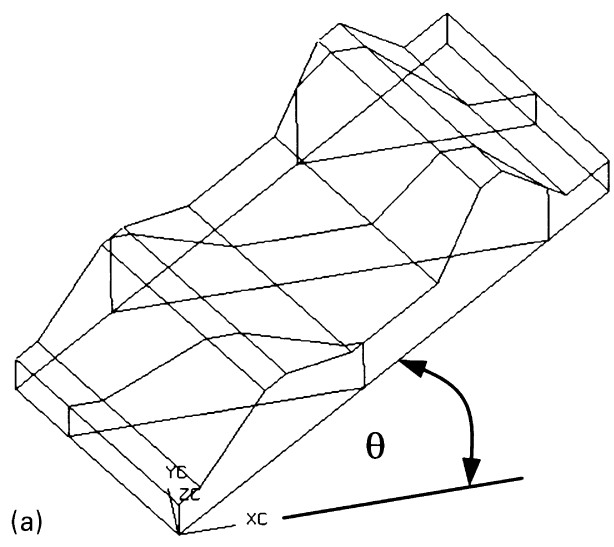

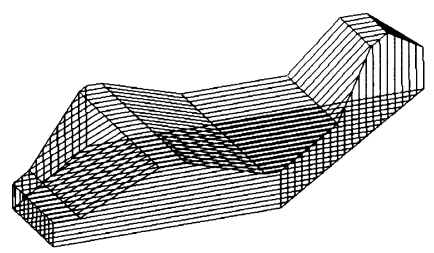

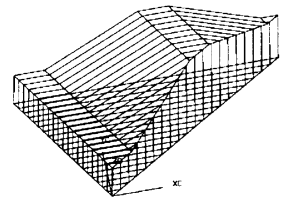

Section 1

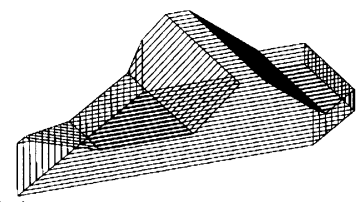

(b) Section 3

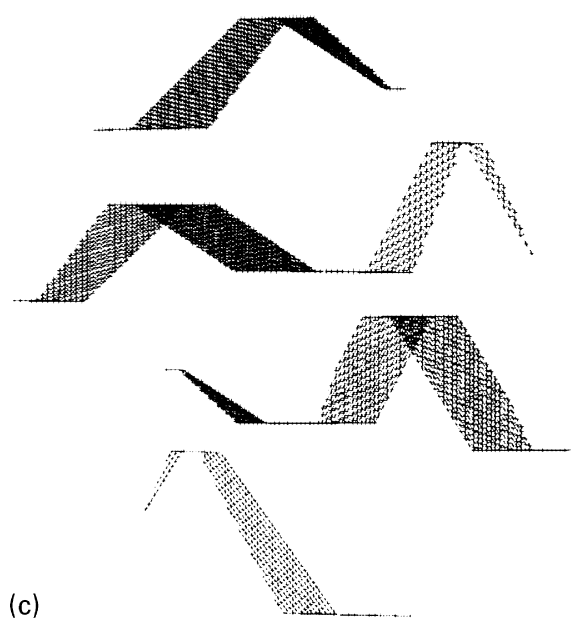

Fig. 3. Slicing process: (a) Step 1; (b) Step 2; (c) Step 3

Having the part sliced, we can determine the surface profile for each section, for the purpose of optimal setting of FOV. Consider a sliced section, the surface profile can be obtained by projecting the surface onto the slicing plane. Connecting all the maximum points of the projection forms the top envelope and that of all the minimum points leads to the bottom envelope. The top and bottom envelopes represent the upper and lower boundary of the section surface, respectively, as shown in Fig. 2(b).

If we set the top of FOV in alignment with the upper surface boundary, it ensures that the top of the surface is always covered, and the variation allowed by the depth of view is fully used to cover the rest in below. We can draw a coverage region by moving the depth of view $D$ with its top following the upper surface boundary. This generates a curve identical to the upper surface boundary with distance $D$ apart. The coverage region is defined by these two curves, as shown in Fig. 2(b) in dotted lines. To examine if the part is fully covered, we can check if the lower surface boundary falls within the coverage region. If answer is yes, then the part is fully covered. Otherwise, it is only partially covered.

Alternatively, one can set the bottom of FOV in alignment with the lower surface boundary. In this case, the surface bottom is guaranteed covered. The coverage region can be obtained by moving the depth of view $D$ with its bottom following the lower surface boundary.

It should be noted that the coverage results are the same for the two settings afore mentioned. In other words, if partially covered by the top setting, it will also be partially covered by the bottom setting, though the non-covered area may be different. The reason is that the depth of view $D$ and the peak-to-valley value of the surface profile are all fixed. This gives rise to the following criteria for checking the coverage:

$D-D_{\mathrm{s}}=\left\{\begin{array}{cc}\geq 0 & \text { fully covered } \\ <0 & \text { partially covered }\end{array}\right.$

where $D_{\mathrm{s}}=z_{\mathrm{p}}-z_{\mathrm{v}}$ represents the peak-to-valley value of the surface profile, $z_{\mathrm{p}}$ and $z_{\mathrm{v}}$ are the peak and valley, respectively. The difference between $D$ and $D_{\mathrm{s}}$ is defined as a coverage margin

$\Delta D=D-D_{\mathrm{s}}$.

A positive coverage margin measures how much it can be maneuvered within FOV while the surface is fully covered. The higher the margin the bigger the tolerance for scanning. A negative margin means that the surface is only partially covered.

\section{Implementation issues}

\subsection{CAD-based slicing}

To implement the proposed path planning method, a CAD-based slicing approach is put forward. For a given part to be scanned the CAD model is sliced into a number of sections. For each sliced section, the surface envelope is determined by discretizing the surface and projecting them onto the slicing plane. This approach is implemented by a $\mathrm{CAD} / \mathrm{CAM}$ package in the following three steps.

Step 1: slicing a CAD model into sections;

Step 2: slicing each section into a set of curves;

Step 3: after projecting the curves to the slicing plane, 

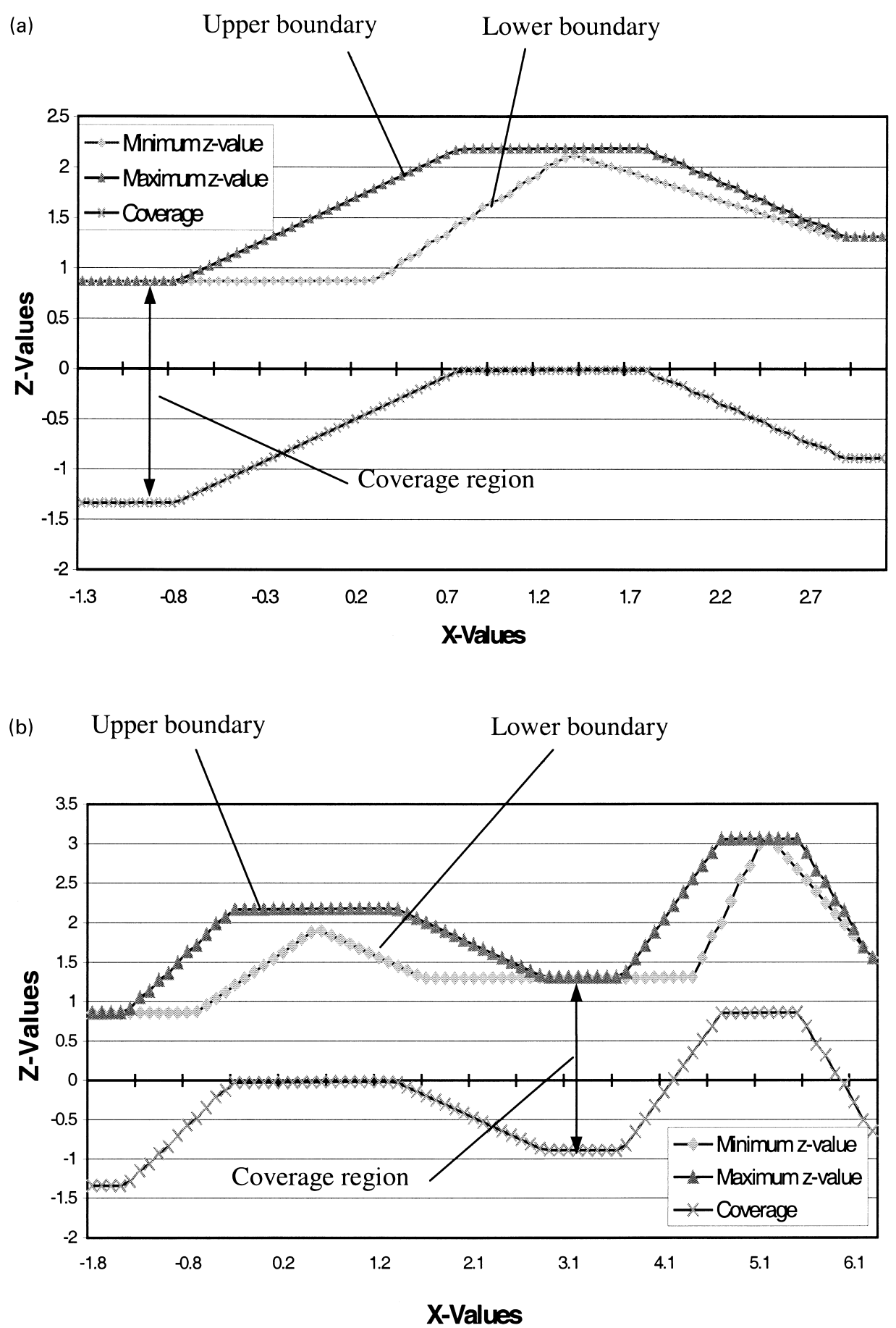

Fig. 4. Scanning path and coverage region of each sliced section: (a) Section 1; (b) Section 2; (c) Section 3; (d) Section 4.

slicing the curves into points to generate the discretized data representing the surface to be scanned.

Fig. 3(a)-(c) show how the sample part is sliced.

\subsection{Path generation}

After slicing, the discretized points representing the surface are used for path generation. By searching for the maximum and minimum points, the upper and lower surface boundary can be determined. By setting the top of FOV along the upper surface boundary, the scanning path for each section is generated. By adding the depth of view, the coverage region is established. To form a complete scanning path, all the paths are connected in a conventional zig-zag fashion.

Due to the limitation in the current motion systems for driving the 3-D line laser scanner, the paths under discussion are line segments. These line segments are created 

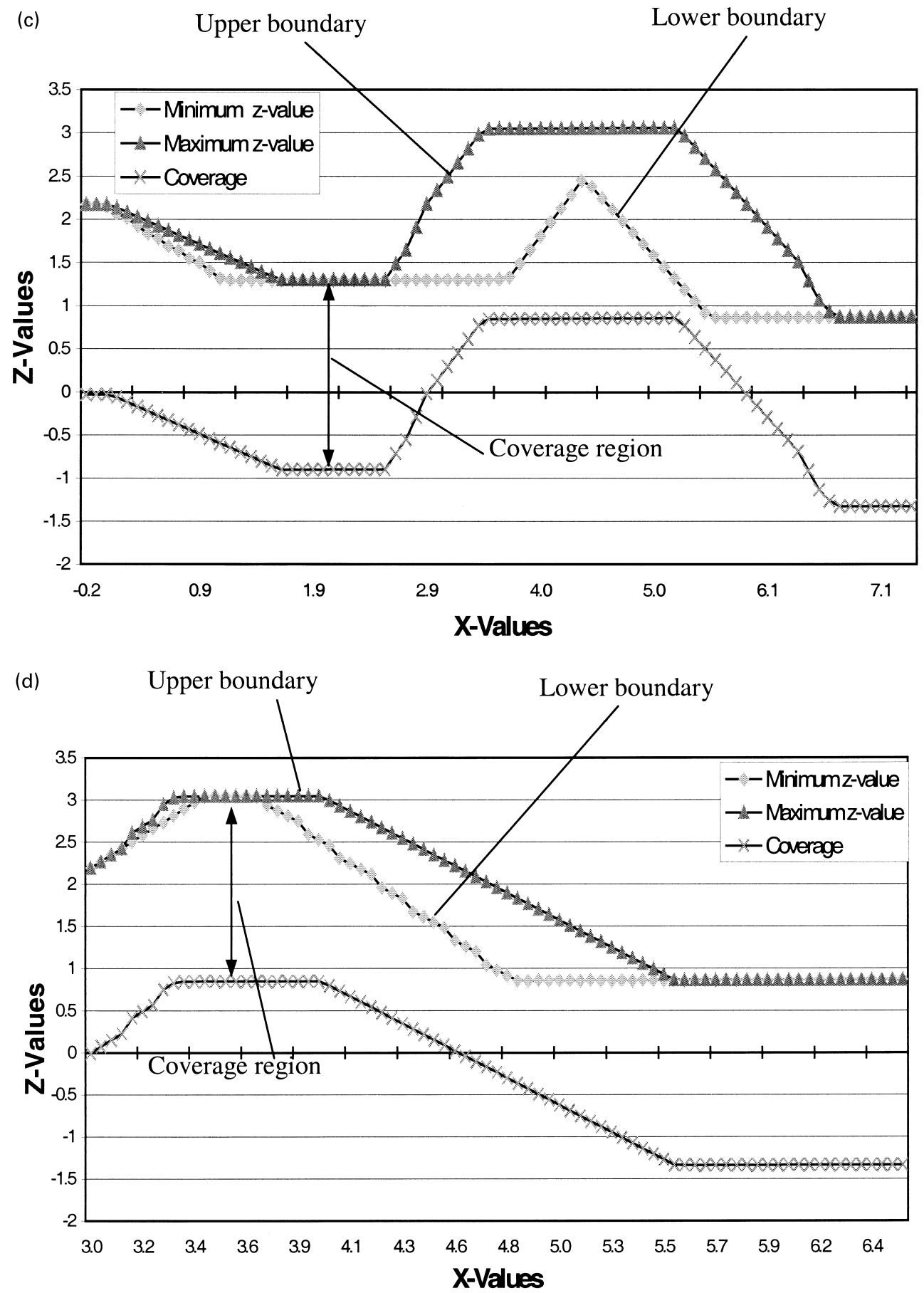

Fig. 4. (continued)

based on the discretized points of the upper boundary curves. The underlying issue is how to link adjacent points. A hybrid method is put forward by combining two common methods, namely the slope method and the bisection method.

The slope method generates line segments by checking the slopes of two adjacent pair of points. If the slopes are within the given tolerance, the adjacent pairs of points are considered along the same line. Otherwise, a new line starts. As for the bisection method it repeatedly divides the data region till the error becomes less than the given tolerance. Suppose a path is defined by $n$ data points, i.e. the data region is $[1, n]$. First, a line is created between the two end points, which is expressed as:

$g(x)=s x+b$ 
Table 1

Effect of part geometry on scanning paths

\begin{tabular}{llll}
\hline$\theta$ & Number of slices & \multicolumn{2}{l}{ Number of line segments } \\
\cline { 3 - 4 } & & Section No. & Segments \\
\hline 0 & 2 & 1 & 9 \\
30 & 4 & 2 & 9 \\
& & 1 & 5 \\
& & 2 & 9 \\
& & 3 & 8 \\
& & 4 & 5 \\
& & 1 & 3 \\
& & 2 & 5 \\
& & 3 & 5 \\
& & 4 & 2 \\
\hline
\end{tabular}

where $s$ and $b$ can be readily obtained as

$s=\left(z_{n}-z_{1}\right) /\left(x_{n}-x_{1}\right)$

$b=z_{n}-s x_{n}$.

In Eqs. 4(a) and (b), $z_{i}$ represents a point along the upper boundary curve with respect to coordinate $x_{i}$ in the scanner's moving direction. The next step is to find the maximum value of error $e_{i}$ between the line and all the data points, i.e.

$e_{i}=\left|z_{i}-\left(s x_{i}+b\right)\right|, \quad$ for $i=1, \ldots, n$.

If the maximum error is within the tolerance, the defined line becomes the desired line segment. If not, for even $n$, interval $[1, n]$ is bisected into two halves, namely, $[1, n / 2]$ and $[n / 2+1, n]$. For odd $n$, two subdivisions will be $[1,(n-1) / 2]$ and $[(n-1) / 2+1, n]$. Bisection terminates when the maximum error is found lower than the given tolerance for all the lines.

Though the slope method can closely follow the discretized points, it often results in a large number of line segments. Especially, in the case that the discretized points are not dense, line segments become very bumpy. As for the bisection method it is straightforward but not efficient. For

Table 2

Effect of part geometry on scanning time and coverage margin

\begin{tabular}{llll}
\hline$\theta$ & \multirow{2}{*}{ Scanning time $(s)$} & \multicolumn{2}{l}{ Coverage margin } \\
\cline { 3 - 4 } & & Section No. & Margin value \\
\hline \multirow{2}{*}{0} & \multirow{2}{*}{133} & 1 & 2.0787 \\
& & 2 & 2.0773 \\
& \multirow{2}{*}{183} & 1 & 1.3017 \\
& & 2 & 0.9596 \\
& & 3 & 0.4160 \\
60 & \multirow{3}{*}{163} & 4 & 1.2188 \\
& & 1 & 0.8910 \\
& & 2 & 1.1637 \\
& & 3 & 0.4523 \\
& & 4 & 0.0078 \\
& & 5 & 1.3201 \\
\hline
\end{tabular}

this reason, the hybrid method is put forward by combining the two methods.

The idea of the hybrid method is to reduce the original data set into a smaller one and then to generate line segments. For a given $n$ data points, first the slope method is used to find all the corner points at which the slopes exceed the given tolerance. No line segments are formed yet. As a result, total $n$ data points will be reduced to the number of the corner points, $n^{\prime}(<n)$.

To generate line segments, Eqs. (3)-(5) of the bisection method are used. However, instead of bisecting, searching is more efficient. The search approach begins with generating a line between the first and last corner point. If the error is over the tolerance, then it moves the second last corner, and so forth, till finding the first line segment. The same process repeats from the end point of the first line segment and the last point.

\section{Experiment}

\subsection{Scanning path}

Refer to the part under study shown in Fig. 3(a), there is an angle $\theta$ defined between the scanner's moving direction $x$ and one of the long edges of the part. As $\theta$ changes the part geometry changes with respect to the scanner's moving direction. Hence, introduction of this angle allows to investigate the effect of part geometry on the scanning path planning. The slicing process shown in Fig. 3(a)-(c) is for $\theta=30^{\circ}$, where four slices are made. Fig. 4 (a)-(d) displays the analysis results of the surface boundaries and the coverage regions for the four slices. In Fig. 4(a)-(d), the upper and lower boundary of each surface are represented by the first curve of maximum $z$ values and the second curve of minimum $z$ values, respectively. The areas formed by these two curves are identical to those shown in Fig. 3(c). The coverage regions are represented by the two curves, one aligned with the upper boundary curve (first curve) and another identical curve below (third curve). In Fig. 4(a)(d), all the lower boundaries (second curves) fall within the coverage regions, and hence the whole surface can be covered.

To consider the effect of the part geometry, two other angles are presented here, $\theta=0$ and $60^{\circ}$. Table 1 summarizes the number of section slices and the number of line segments for the three angles. It can be seen that change in the part geometry causes change in the number of sliced sections and consequently the number of line segments of the paths. These changes also affect the coverage margins and the time spent on scanning, as given in Table 2.

\subsection{Scanning tests}

A Hymarc 3-D line laser scanner is used for scanning tests. The scanner is mounted on a Brown and Sharpe 
(a)
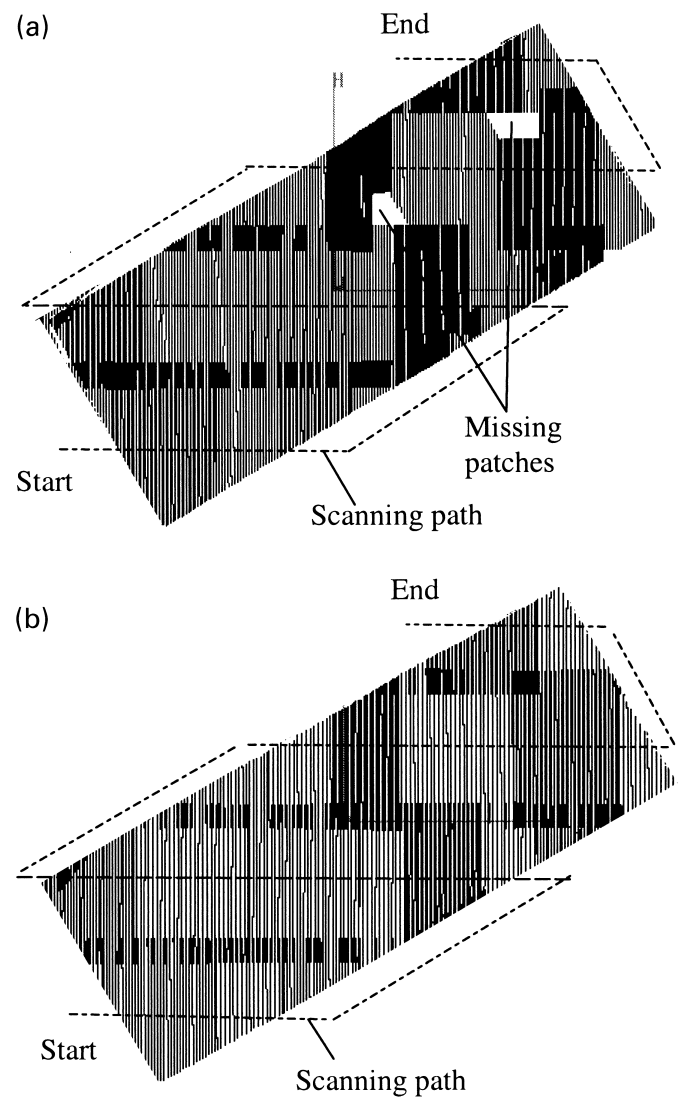

Fig. 5. Scanning process: (a) Incomplete (top view); (b) Complete (top view).

CMM. For scanning implementation, the scanning paths are generated by an in-house program developed based on the method described before. The path files are then converted into AVAIL programs for driving the Brown and Sharpe CMM. Since the path programs are created with respect to the part coordinate system as shown in Fig. 3(a), the CMM's coordinates need to be aligned with the part coordinates. This part alignment is currently done manually which introduces some error. This error may affect the image data collection where the coverage margin is small. Once the initial alignment is done, the entire scanning process is automated.

Fig. 5 show two scan images for $\theta=30^{\circ}$. Fig. 5(a) is the first scan that is incomplete. The data missing area occurs at the third section, where the coverage margin is small, only 0.4160 , and cannot offset the alignment error. Hence further adjustment is required. Fig. 5(b) shows the second scan after adjustment and it is complete.

\section{Conclusions}

A path planning method is developed for the 3-D line laser scanner. The method considers how to set the field of view of the scanner in order to achieve a maximum coverage. The proposed method utilizes a CAD/CAM package for determining the surface profile and based on that to generate paths for driving the laser scanner. Implementation issues are discussed including the effect of the part geometry and part alignment. It can be seen that with the proposed method a 3-D line laser scanning process can be effectively automated.

\section{Acknowledgements}

The authors would like to thank Stan Kowala of the National Research Council of Canada, Bert VandenBerg of Hymarc for their help in this work.

\section{References}

[1] Whohlers T. 3D digitizing for engineering. Computer Graphics 1995;March:1-3.

[2] DeYoung HG. The technology at work. Computer Graphics 1995;March:14-16.

[3] Milroy MJ, Weir DJ, Bradley C, Vickers GW. Reverse engineering employing a 3-D laser scanner: a case study. The International Journal of Advanced Manufacturing Technology 1996;12:111-121.

[4] Cheng WL, Menq CH. Integrated laser/CMM system for the dimensional inspection of objects made of soft materials. The International Journal of Advanced Manufacturing Technology 1995;10:35-45.

[5] Newman TS, Jain AK. A survey of automated visual inspection. Computer Vision and Image Understanding 1995;61(2):231-262.

[6] Smith KB, Zheng YF. Accuracy analysis of point laser triangulation probes using simulations. ASME Journal of Manufacturing Science and Engineering 1998;120:736-745.

[7] Rioux M, Bechthold G, Taylor D, Duggan M. Design of a large depth of view three dimensional camera for robot vision. Optical Engineering 1987;26:1245-1250.

[8] Baribeau R, Rioux M. Influence of speckle on laser range finders. Applied Optics 1991;30(30):2873-2878.

[9] Milroy MJ, Bradley C, Vickers GW. Automated laser scanning based orthogonal cross section. Machine Vision and Applications 1996;9:106-118.

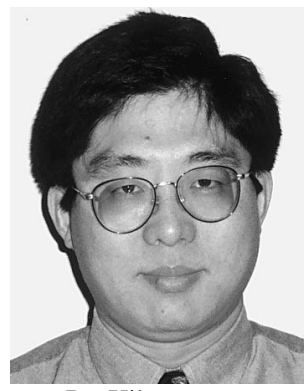

Fengfeng Xi received his $\mathrm{PhD}$ degree from the Department of Mechanical Engineering of University of Toronto in 1993. Upon graduation, he was awarded a Canadian NSERC postdoctoral fellowship with which he worked in the Center for Intelligent Machines of McGill University and the Pattern Analysis and Machine Intelligence Laboratory of University of Waterloo. He joined the National Research Council of Canada in 1994, first with the Institute for Sensors and Controls, and now with the Integrated Manufacturing Technologies Institute. Dr. Xi's current research interests are in the area of intelligent production systems, including laser scanning.

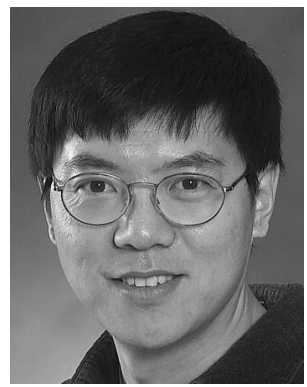

Chang Shu is a research scientist at the National Research Council of Canada. He received BSc from Habrin Institute of Technology, China, in 1985, and $\mathrm{PhD}$ in computer science, from Queen Mary and Westfield College, University of London, UK, in 1992. His research interests include geometric modeling, computational geometry, and their applications in CAD/CAM, robotics, graphics, and $3 \mathrm{D}$ imaging. 